\title{
Pleuroparenchymal fibroelastosis from a consecutive database: a rare disease entity?
}

\author{
To the Editor:
}

Pleuroparenchymal fibroelastosis (PPFE) is a rare condition characterised by predominantly upper lobe involvement with pleural fibrosis and subjacent parenchymal fibroelastosis [1, 2]. Idiopathic PPFE (IPPFE), which was included in the rare idiopathic interstitial pneumonias (IIPs) in the update of the international multidisciplinary classification of IIPs published in 2013 [3], was first described in the Japanese literature by AMITANi et al. in 1992 [4]. PPFE has been reported to be associated with drugs, chronic hypersensitivity pneumonia, collagen vascular diseases, infections, and bone marrow transplantations [5-8]. However, the frequency of PPFE occurrence has been uncertain. We hypothesised that PPFE was not a rare disease entity and conducted this study. Informed consent was obtained from all patients, and the Institutional Review Board of the National Hospital Organization Kinki-Chuo Chest Medical Center (KCCMC) (Sakai City, Japan) approved this study.

To prove the hypothesis, we retrospectively reviewed our database of diagnostic pathology archives from 205 consecutive patients who had undergone a surgical lung biopsy (SLB) to diagnose diffuse lung diseases at the KCCMC from 2004 to 2012, and selected 14 cases in which the key words "atelectatic fibrosis", "pleuroparenchymal fibroelastosis" or "parenchymal fibroelastosis" were identified. The 14 cases were re-evaluated in multidisciplinary discussions using histopathological and radiological criteria for the diagnosis of PPFE $[1,2,5]$. Two cases were excluded from a diagnosis of PPFE; one did not fulfil the radiological criteria and the other showed a pathological pattern of usual interstitial pneumonia (UIP), with only focal elastosis.

We evaluated the pathology according to the report by REDDY et al. [5]. Patients with upper zone subpleural elastosis, intra-alveolar collagen deposition and pleural thickening with fibrosis were categorised as definite PPFE. Patients without pleural thickening were categorised as consistent with PPFE. For the radiological findings from high-resolution computed tomography (HRCT) pleural thickening associated with subpleural fibrosis was observed mainly in the upper lobes, with less marked or no involvement of the lower lobes. Features suggesting comorbid disease were allowed to be present elsewhere in the lung. Pathological and radiological patterns of interstitial lung diseases (ILDs) were determined by the criteria previously reported $[3,9,10]$. A radiological pattern of nonspecific interstitial pneumonia (NSIP) was defined according to a previous report [11].

Of the 205 consecutive patients who had undergone a SLB for ILDs, 12 (5.9\%) cases were identified as PPFE after multidisciplinary discussion (definite PPFE: eight cases; consistent with PPFE: four cases). In the same period, the number of patients presenting ILDs on HRCT and receiving bronchoalveolar lavage was 1622, including the $205(12.6 \%)$ cases with SLB. Of the 205 cases, 77 cases were diagnosed as IIPs. Clinical characteristics of the PPFE cases are summarised in table 1. Seven (58\%) were male and the median age was 62 years old. Seven (58\%) had never-smoked. Eight (67\%) developed spontaneous pneumothoraces during the course of their disease and six (50\%) experienced pneumothoraces repeatedly. Eight (3.9\%) patients undergoing SLB were categorised as IPPFE, while four (2.0\%) were categorised as secondary PPFE (SPPFE). Thus, the frequency of IPPFE was not rare among IIPs (10.4\%).

The HRCT findings of patients with PPFE are summarised in table 1 . All 12 patients revealed bilateral irregular pleuroparenchymal thickening in the upper zone. 10 demonstrated elevated hilar shadows suggesting volume reduction in the upper lobes. Notably, 11 (92\%) demonstrated coexistent ILD in the lower lobes (UIP pattern: five cases; possible UIP pattern: four cases; NSIP pattern: one case; and an undefined pattern: one case).

Follow-up HRCT images were available for 10 patients (follow-up period: 13-69 months, median 22.5 months). Of these patients, seven (70\%) demonstrated progression of pleuroparenchymal thickening, and four (40\%) had increased or newly apparent honeycombing at their last HRCT. In seven (70\%) out of the 10 discrete cysts increased or enlarged. In one of these cases, a ball of fungus appeared in the upper lobe cyst.

For pathological findings, 10 patients underwent SLBs in the upper lobes, with the remaining two undergoing biopsies in the middle lobes. All patients exhibited subpleural elastosis and intra-alveolar 
TABLE 1 Summary of the 12 cases of pleuroparenchymal fibroelastosis (PPFE)

\begin{tabular}{|c|c|}
\hline $\begin{array}{l}\text { Subjects } \mathrm{n} \\
\text { Idiopathic/secondary PPFE } \mathrm{n} / \mathrm{n}^{\#} \\
\text { Age years }\end{array}$ & $\begin{array}{c}12 \\
8 / 4 \\
62(27-70)\end{array}$ \\
\hline \multicolumn{2}{|l|}{ Sex } \\
\hline Males & 7 (58) \\
\hline Females & $5(42)$ \\
\hline BMI $\mathrm{kg} \cdot \mathrm{m}^{-2 \uparrow}$ & $20.0(13.5-24.2)$ \\
\hline \multicolumn{2}{|l|}{ Smoking habits ? } \\
\hline Never-smoker & 7 (58) \\
\hline Current or ex-smoker & $5(42)$ \\
\hline \multicolumn{2}{|l|}{ Clinical symptoms ${ }^{\pi}$} \\
\hline Asymptomatic & $3(25)$ \\
\hline Symptomatic & 9 (75) \\
\hline Dyspnoea on exertion & $7(58)$ \\
\hline Dry cough & $5(42)$ \\
\hline Chest pain & $1(8)$ \\
\hline Pneumothorax & $8(67)$ \\
\hline \multicolumn{2}{|l|}{ HRCT findings $^{+}$} \\
\hline Pleuroparenchymal thickening & $12(100)$ \\
\hline Elevated hilar shadows & $10(83)$ \\
\hline Coexistent ILD in lower lobes & 11 (92) \\
\hline UIP pattern & $5(42)$ \\
\hline Possible UIP pattern & $4(33)$ \\
\hline NSIP pattern & $1(8)$ \\
\hline Others & $1(8)$ \\
\hline \multicolumn{2}{|l|}{ Pathological findings } \\
\hline Subpleural elastosis & $12(100)$ \\
\hline Intra-alveolar collagen deposition & $12(100)$ \\
\hline Pleural thickening with fibrosis & $8(67)$ \\
\hline Preserved alveolar structure & $12(100)$ \\
\hline Coexistent ILD in lower lobes & $9(75)$ \\
\hline UIP pattern & $8(67)$ \\
\hline Non-classifiable interstitial pneumonia pattern & $1(8)$ \\
\hline \multicolumn{2}{|l|}{ Pulmonary function test $(n=8)$} \\
\hline FVC $\%$ predicted $^{\text {I }}$ & $70.6(53.8-108.6)$ \\
\hline FVC decline $\mathrm{mL} \cdot$ year $^{-1}$ & $-187(-878-4)$ \\
\hline TLC \% predicted ${ }^{\Uparrow}$ & $71.7(50.3-133.9)$ \\
\hline TLC decline $\mathrm{mL} \cdot \mathrm{year}^{-1}$ & $-310(-1335--54)$ \\
\hline \multicolumn{2}{|l|}{ Medication } \\
\hline Steroid & $6(50)$ \\
\hline Immunosuppressant & 7 (58) \\
\hline Pirfenidone & $4(33)$ \\
\hline \multicolumn{2}{|l|}{ Median survival time days } \\
\hline From the first visit & $2459(55-2996)$ \\
\hline From SLB & 838 (29-2014) \\
\hline
\end{tabular}

Data are presented as median (range) or $\mathrm{n}(\%)$ unless otherwise stated. BMI: body mass index; HRCT: high-resolution computed tomography; ILD: interstitial lung disease; UIP: usual interstitial pneumonia; NSIP: nonspecific interstitial pneumonia; FVC: forced vital capacity; TLC: total lung capacity; SLB: surgical lung biopsy. \#: of the four secondary PPFE cases one was a cyclophosphamide-induced case and the remaining three cases coexisted with chronic hypersensitivity pneumonia, rheumatoid arthritis or Sjögren's syndrome; ${ }^{\text {I: }}$ the data were obtained at the first visit; ${ }^{+}$: the data were obtained before the SLB.

collagen deposition; however, four (33\%) did not have pleural thickening with fibrosis. Notably, nine (75\%) showed coexistent ILDs (eight with UIP pattern and one with non-classifiable interstitial pneumonia pattern) (table 1).

Pulmonary function test data were available for eight patients without the complication of pneumothoraces. The baseline levels of median forced vital capacity (FVC) and total lung capacity (TLC) were 70.6 and $71.7 \%$ predicted, respectively. There was a marked decline in FVC and TLC.

Eight patients received medication. Six were treated with low-dose corticosteroids. Four out of these six patients received additional immunosuppressant therapy (cyclosporine $(n=2)$ and azathioprine $(n=2))$. 
The other two of these six patients received both immunosuppressant therapy (azathioprine) and pirfenidone. Of the remaining two patients, one received both immunosuppressant therapy (azathioprine) and pirfenidone, while the other was treated only with pirfenidone (table 1). However, we did not find any improvement in pulmonary function tests, HRCT or symptoms.

The median survival time for PPFE patients from the first hospital visit and from the SLB were 2459 days and 838 days, respectively. The survival from SLB seemed to be poor. The number of patients was too small to tell the difference between the survival time for IPPFE and SPPFE patients, and of those with definite PPFE and consistent with PPFE. Seven patients were alive at the last follow-up (four of whom required home oxygen therapy), while the remaining five died from respiratory failure due to disease progression.

Although fibrotic thickening of the pleura is one of the features of PPFE patients [1], four patients (three IPPFE and one cyclophosphamide-induced case) did not have pleural thickening in the present study. These four cases were diagnosed as consistent with PPFE. The three IPPFE cases among these four cases did not have radiotherapy, chemotherapy or inhalational injuries, which are known aetiologies of intra-alveolar fibrosis with septal elastosis. Some reports have included PPFE cases without pleural thickening $[4,5,8,12,13]$. Further studies are required to clarify the significance of pleural thickening in the diagnosis of PPFE.

Patients with a coexistent ILD other than fibroelastosis were observed in the present study as previously reported [5, 12-14]. The frequency of such cases was $75 \%$ in our report and might be higher than the report by REDDY et al. [5] (43\%); however, the patients in the other reports did not always accept SLB of the lower lobe. WATANABE et al. [13] mentioned eight out of nine cases of idiopathic upper lobe fibrosis, which is similar to PPFE, showed lower lobe lesions on HRCT although they were not pathologically evaluated. Thus, coexistent ILD is supposedly more frequently observed when histologically examined.

In pulmonary function tests FVC declined rapidly in most patients. In the report by WATANABE et al. [13] respiratory function also declined remarkably in PPFE patients. These findings suggest that PPFE is a progressive disease. However, in the current study drug therapies including pirfenidone were not effective. Given this, at present, lung transplantation would be the only effective treatment for PPFE.

From these findings, IPPFE is an acceptable disease entity among IIPs, and is not as rare as previously reported. PPFE is a progressive disease with the frequent complication of pneumothorax and there is no effective therapy. Development of effective anti-fibrotic and/or anti-elastotic treatment is required.

@ERSpublications

Idiopathic pleuroparenchymal fibroelastosis is an acceptable entity among IIPs and not as rare as previously reported http://ow.ly/I5NEz

Takeshi Nakatani ${ }^{1}$, Toru Arai $^{2}$, Masanori Kitaichi ${ }^{2,3}$, Masanori Akira ${ }^{2,4}$, Kazunobu Tachibana ${ }^{1,2}$, Chikatoshi Sugimoto $^{2}$, Aya Hirooka ${ }^{1}$, Taisuke Tsuji ${ }^{1}$, Shojiro Minomo ${ }^{1}$, Seiji Hayashi ${ }^{1}$ and Yoshikazu Inoue ${ }^{2}$

${ }^{1}$ Dept of Internal Medicine, National Hospital Organization Kinki-Chuo Chest Medical Center, Sakai City, Japan. ${ }^{2}$ Clinical Research Center, National Hospital Organization Kinki-Chuo Chest Medical Center, Sakai City, Japan. ${ }^{3}$ Dept of Pathology, National Hospital Organization Kinki-Chuo Chest Medical Center, Sakai City, Japan. ${ }^{4}$ Dept of Radiology, National Hospital Organization Kinki-Chuo Chest Medical Center, Sakai City, Japan.

Correspondence: Yoshikazu Inoue, Clinical Research Center, National Hospital Organization Kinki-Chuo Chest Medical Center, 1180 Nagasone-Cho, Kita-Ku, Sakai, Osaka 591-8555, Japan. E-mail: giichi@kch.hosp.go.jp

Received: Sept 262014 | Accepted after revision: Jan 112015 | First published online: Feb 192015

Support statement: This study was partially supported by a grant from the Japanese Ministry of Health, Labour, and Welfare (grant numbers: 14427648, 14526278 and 14526182) (to Y. Inoue) and from the National Hospital Organization, Japan, Respiratory network grant (to Y. Inoue and T. Arai).

Conflict of interest: None declared.

\section{References}

1 Frankel SK, Cool CD, Lynch DA, et al. Idiopathic pleuroparenchymal fibroelastosis: description of a novel clinicopathologic entity. Chest 2004; 126: 2007-2013.

2 Becker CD, Gil J, Padilla ML. Idiopathic pleuroparenchymal fibroelastosis: an unrecognized or misdiagnosed entity? Mod Pathol 2008; 21: 784-787.

3 Travis WD, Costabel U, Hansell DM, et al. An official American Thoracic Society/European Respiratory Society statement: update of the international multidisciplinary classification of the idiopathic interstitial pneumonias. $\mathrm{Am}$ J Respir Crit Care Med 2013; 188: 733-748.

4 Amitani R, Niimi A, Kuze F. [Idiopathic pulmonary upper lobe fibrosis (IPUF).] Kokyu 1992; 11: 693-699.

5 Reddy TL, Tominaga M, Hansell DM, et al. Pleuroparenchymal fibroelastosis: a spectrum of histological and imaging phenotypes. Eur Respir J 2012; 40: 377-385. 
6 von der Thüsen JH, Hansell DM, Tominaga M, et al. Pleuroparenchymal fibroelastosis in patients with pulmonary disease secondary to bone marrow transplantation. Mod Pathol 2011; 24: 1633-1639.

7 Beynat-Mouterde C, Beltramo G, Lezmi G, et al. Pleuroparenchymal fibroelastosis as a late complication of chemotherapy agents. Eur Respir J 2014; 44: 523-527.

8 Camus $\mathrm{P}$, von der Thüsen J, Hansell DM, et al. Pleuroparenchymal fibroelastosis: one more walk on the wild side of drugs? Eur Respir J 2014; 44: 289-296.

9 American Thoracic Society, European Respiratory Society. American Thoracic Society/European Respiratory Society International Multidisciplinary Consensus Classification of the Idiopathic Interstitial Pneumonias. Am J Respir Crit Care Med 2002; 165: 277-304.

10 Raghu G, Collard HR, Egan JJ, et al. An official ATS/ERS/JRS/ALAT statement: idiopathic pulmonary fibrosis: evidence-based guidelines for diagnosis and management. Am J Respir Crit Care Med 2011; 183: 788-824.

11 Akira M, Inoue Y, Kitaichi M, et al. Usual interstitial pneumonia and nonspecific interstitial pneumonia with and without concurrent emphysema: thin section CT findings. Radiology 2009; 251: 271-279.

12 Oda $\mathrm{T}$, Ogura $\mathrm{T}$, Kitamura $\mathrm{H}$, et al. Distinct characteristics of pleuroparenchymal fibroelastosis with usual interstitial pneumonia compared with idiopathic pulmonary fibrosis. Chest 2014; 146: 1248-1255.

13 Watanabe K, Nagata N, Kitasato $\mathrm{Y}$, et al. Rapid decrease in forced vital capacity in patients with idiopathic pulmonary upper lobe fibrosis. Respir Investig 2012; 50: 88-97.

14 Shiota S, Shimizu K, Suzuki M, et al. [Seven cases of marked pulmonary fibrosis in the upper lobe.] Nihon Kokyuki Gakkai Zasshi 1999; 37: 87-96. 